Research Paper

\title{
Ets2 knockdown inhibits tumorigenesis in esophageal squamous cell carcinoma in vivo and in vitro
}

\author{
Qinghua $\mathrm{Li}^{1,2, *}$, Lu Yang ${ }^{2, *}$, Kang Han ${ }^{2, *}$, Liqiang Zhu ${ }^{3}$, Yanting Zhang ${ }^{2}$, Shanshan \\ $\mathrm{Ma}^{2}$, Kun Zhang ${ }^{2}$, Bo Yang ${ }^{1}$, Fangxia Guan ${ }^{1,2}$ \\ ${ }^{1}$ The First Affiliated Hospital of Zhengzhou University, Zhengzhou 450052, Henan Province, China \\ ${ }^{2}$ School of Life Sciences, Zhengzhou University, Zhengzhou 450001, Henan Province, China \\ ${ }^{3}$ The Second Affiliated Hospital of Zhengzhou University, Zhengzhou 450002, Henan Province, China \\ *These authors contributed equally to this work
}

Correspondence to: Fangxia Guan, email: guanfangxia@126.com Bo Yang, email: yangbo96@126.com

Keywords: Ets2, esophageal squamous cell carcinoma, proliferation, apoptosis, mTOR/p70S6K signaling pathway

Received: December 10, 2015

Accepted: August 08, 2016

Published: August 18, 2016

\begin{abstract}
Increased expression of Ets 2 is reported upregulated in esophageal squamous cell carcinoma tissue. However, the function of Ets 2 in carcinogenesis of ESCC is poorly understood. Here, the rise of Ets 2 was confirmed in ESCC cells and Ets 2 depletion by RNA interference promotes cell apoptosis, inhibits cell proliferation, attenuates cell invasion and induces cell cycle G0/G1 arrest in vitro. Moreover, in vivo, Xenograft mouse model studies showed Ets2 knockdown inhibits tumor formation and metastasis significantly. Furthermore, Ets 2 depletion inactivates the mTOR/p70S6K signaling pathway both in vitro and in vivo. Taken together, these findings strongly suggest that a critical role of Ets 2 in human ESCC pathogenesis via the inactivation of the $\mathrm{mTOR} / \mathrm{p} 7056 \mathrm{~K}$ signaling pathway.
\end{abstract}

\section{INTRODUCTION}

Esophageal squamous cell carcinoma (ESCC) is one of the most frequently diagnosed cancers in developing countries, especially in Northern China [1]. Although therapeutic strategies have improved, the prognosis of patients with ESCC is still poor owing to early invasion and metastasis and the 5-year survival rate of this disease after surgery and radiation therapy is only $10 \sim 20 \%$ [2]. The dismal outcome of ESCC is attributed to the largely unknown molecular mechanism in tumorigenesis and progression. In order to ultimately reduce morbidity and mortality of the disease, a greater understanding of the molecular events underlying carcinogenesis, progression and metastasis of ESCC is required.

The Ets was originally discovered as part of the gagmyb-ets transforming fusion protein of an avian replicationdefective retrovirus, E26 [3], and the Ets family has been established as one of the largest families of transcriptional regulators, with diverse functions and activities. To date, 28 human Ets family members have been identified [3]. All the Ets transcription factors comprise a large evolutionarily conserved gene family characterized by the Ets domain, and this 85 amino acid region forms the winged helix-turn-helix DNA binding domain composed of three alpha helices and a four-stranded beta sheet. The Ets protein controls gene expression by binding to numerous genes with GGA (A/T) Ets binding site (EBS) [4] and over 400 Ets target genes have been defined based upon the presence of functional EBS in their regulatory regions [3], thus the Ets transcription factors impact a broad spectrum of cellular functions including proliferation, cell cycle, differentiation, migration, transformation, and apoptosis [5-8]. Ets2 is one of the founder members of the Ets family located on human chromosome 21 and was initially characterized as a proto-oncogene. Multiple studies with cell lines and animal models suggest that Ets2 causally exhibit both tumor-promoting and tumor-suppressive effects in distinct carcinomas. For example, Ets2 amplification has also been demonstrated in patients with acute nonlymphoblastic leukaemia [9], and Ets2 is expressed at elevated levels in breast, ocular neoplasm, cervical and prostate cancer [10-13]. However, Kabbout et al. revealed marked decline of Ets2 transcript expression in non-small cell lung cancer compared with paired normal lung tissues [14], exhibiting the tumor-suppressive effects of Ets2. The tumorpromoting/-suppressive effects of Ets2 might depend on the protein expression with potentially important functions 
in the tumor microenvironment, including growth factors, adhesion molecules, extracellular proteases and antiapoptotic genes. Ets2 is a downstream target for both the Ras/Raf/MAP kinase and phosphatidylinositol 3-kinase/ Akt pathways $[15,16]$, regulating the expression of a number of genes with potentially important functions in the tumor microenvironment.

Although Li et al. observed that Ets2 rose $75.7 \%$ $(28 / 37)$ at mRNA level and 75\% (12/16) at protein level in ESCC tissues relative to matched normal tissues [18], little work has been carried out at the molecular and cellular mechanisms of Ets 2 action. Herein, we report on the results of Ets2 knockdown mediated by siRNA fragments and lentivirus on apoptosis, invasion, et al. and the signaling Ets2 involved in ESCC both in vitro and in vivo.

\section{RESULTS}

\section{Overexpressed Ets2 is depleted in the ESCC cell lines}

Firstly, we confirmed whether Ets2 expression level was elevated in ESCC cells by Western blotting and the results demonstrated that the Ets2 expression was increased by 3.2-fold in EC9706 cells $(P<0.01), 2.2$-fold in Eca109 cells $(P<0.05)$, and 1.93 -fold in EC1 cells $(P<0.05)$ respectively compared with that in Het-1A cells (Figure 1A and 1B). And, the expression of Ets2 protein in EC9706 cells was higher than that in Eca109 and EC1 cells $(P<0.05)$.

To better understand the role of Ets 2 in ESCC, three candidate siRNA fragments again Ets2 (marked as siRNA1, siRNA2, siRNA3) were synthesized to interfere Ets2 expression and the recombinant siRNA particles were transfected into ESCC cells with Lipofectamine ${ }^{\circledR}$ 2000 Reagent (Invitrogen) following the manufacturer's protocol. ESCC cells transfected with equal amounts of Lipofectamine ${ }^{\circledR} 2000$ Reagent (lip2000) were used to eliminate the influence of the transfection reagent, ESCC cells were cultured as control (CON) and ESCC cell were transfected with non-targeting control siRNA as negative control (NC). As shown in Figure 1C and 1D, Ets2 was significantly decreased compared with $\mathrm{NC}$ and $\mathrm{CON}$ only at $48 \mathrm{~h}$ after transfection with siRNA1 and siRNA2 fragments in EC9706 cells. And as shown in Figure 1E, the interference efficiency of siRNA1 fragments was significantly higher than that of siRNA2 and siRNA3. Thus the siRNA1 sequence against Ets2 was chosen to knock Ets2 down and the optimal time for observation was at $48 \mathrm{~h}$ after transfection.

\section{Ets2 knockdown suppresses ESCC cells proliferation in vitro and in vivo}

We next investigated the effects of Ets2 depletion on cell proliferation and survival by 5-ethynyl-2'deoxyuridine (EdU) assay and CCK-8. The Edu assay revealed that the proliferative rates of EC9706, Eca109 and EC1 cells worked out $19.97 \%, 29.2 \%$ and $22.3 \%$ respectively after Ets2 depletion, reducing by $40 \%$, $45 \%$ and $57 \%$ compared with NC cells (Figure 2A). Meanwhile, detection of cell viability of ESCC cells by CCK-8 showed that the cell survival rates of EC9706, Eca109 and EC1 cells were $69 \%, 58 \%$ and $68 \%$ after transfection with siEts2, reducing by $31 \%, 42 \%$ and $32 \%$ compared with NC (Figure 2B), respectively. Additionally, the proliferative rates and cell survival rates changed without differences among NC, CON and lip2000 groups. These data suggest that the Ets2 decrease could suppress the proliferation of ESCC cells in vitro.

In order to confirm whether the growth-inhibiting effect of Ets2 depletion is relevant to ESCC growth in vivo, silent expression of Ets2 were inoculated into $\mathrm{BALB} / \mathrm{C}$ athymic mice after siRNA1 sequence was ligated into retroviral vectors to establish the Eca109 cell stably silencing Ets2. Silencing Ets2 expression inhibited tumor growth (Figure 7A) and induced a decrease in tumor weight and volume (Figure 7B). Taking all together, we concluded that Ets2 inhibits the proliferation of ESCC cells in vivo and in vitro.

\section{Ets2 depletion promotes apoptosis of ESCC cells in vitro and in vivo}

Apoptotic cell death of ESCC cells was examined using flow cytometric analysis staining with FITCconjugated annexin- $\mathrm{V}$ as an early marker for apoptosis. Cells were simultaneously stained with PI to investigate loss of cell membrane integrity. This double staining procedure distinguishes early stage apoptotic cells (annexin V-positive) from late stage apoptotic cells (annexin V-positive, PI positive). As shown in Figure 3A, when Ets2 was knocked down in EC9706 cells, the percentages of early and late stage apoptotic cells were increased from $1.9 \%$ and $0.9 \%$ in $\mathrm{NC}$ to $6.8 \%$ and $5.1 \%$ respectively $(P<0.05)$. Early and late stage apoptotic cells in Eca109 cells were increased from $6.6 \%$ in NC to $11.7 \%$ and from $0.3 \%$ in $\mathrm{NC}$ to $1.1 \%$ respectively $(P<0.05)$. However, only the late stage apoptotic cells were increased from $2.8 \%$ to $7.3 \%(P<0.05)$ in Ets2-depleted EC1 cells. Furthermore, Tunel assay showed that apoptotic rate was $17.4 \%$ in Ets2 depleted tumor tissue (Figure 8).

In addition, the cell apoptosis factor caspase-3 and Bcl-2 were detected in our study in vitro and in vivo. The results of Western blotting revealed cell apoptosis factor caspase- 3 was increased dramatically compared to their corresponding control cells and tissue (Figure 3B and D, $P<0.05)$. In contrast, anti-apoptotic Bcl-2 protein was decreased when Ets2 was knocked-down (Figure 7C and 7D, $P<0.001)$. Taken together, these results indicated that early stage apoptosis was induced by depletion of Ets2 in ESCC cells in vitro and in vivo. 
A
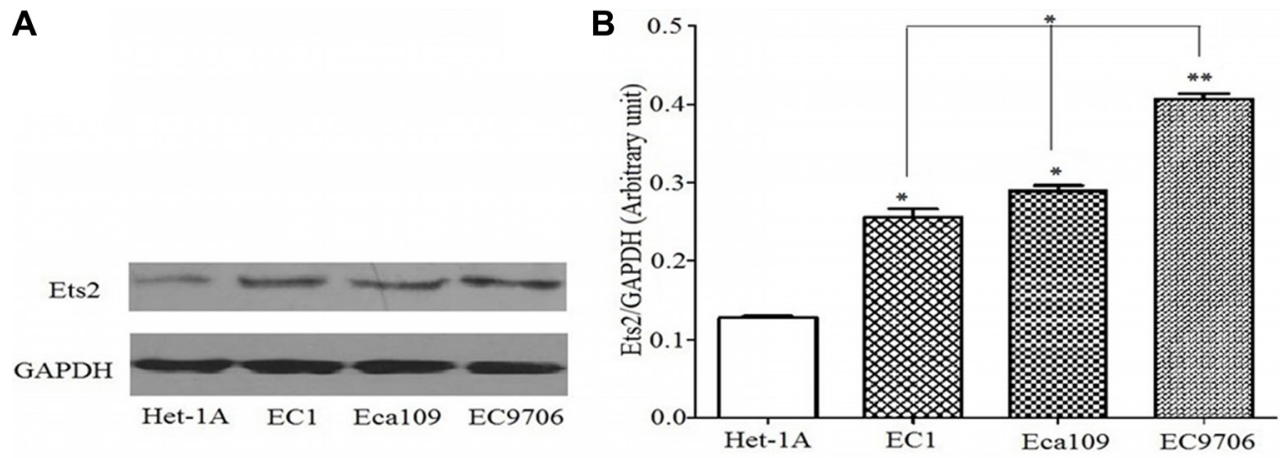

C

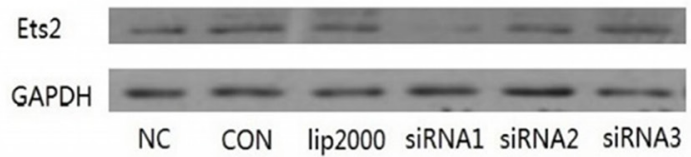

D

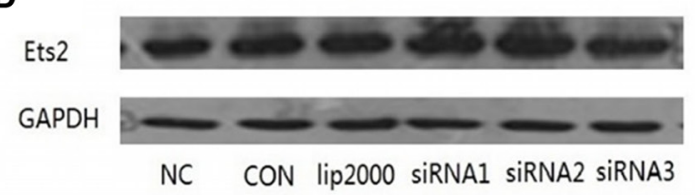

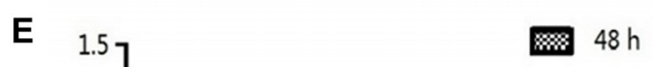

$\left.{ }^{1.5}\right] \quad$ m $48 \mathrm{~h}$

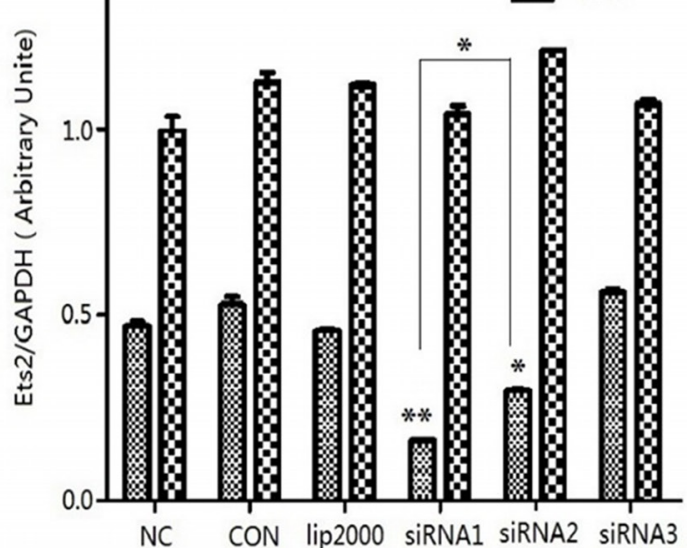

Figure 1: Expression of Ets2 protein was notably increased in ESCC cells and knocked down by siRNA. (A) Western Blot analysis of Ets2 in Het-1 A, EC1, EC9706 and Eca109 cells. (B) Semi-quantitative analysis showed that Ets2 was increased by 3.2-fold in EC9706 cells $(P<0.01), 2.2$-fold in Eca109 cells $(P<0.05)$, and 1.93 -fold in EC1 cells $(P<0.05)$ respectively compared with that in Het-1A cells. $* P<0.05, * * P<0.01$ versus that in Het-1A cells. (C) and (D) Western blotting analysis of the expression of Ets2 in EC9706 cells at $48 \mathrm{~h}$ and $72 \mathrm{~h}$ after transfection with 3 candidate siRNA sequences (marked as siRNA1, siRNA2, siRNA3). (E) Quantitative results of the Western blotting analysis obtained via densitometric analysis. Ets2 protein expression was obviously inhibited by interference only with siRNA1 and siRNA2 $(P<0.01)$ sequences at $48 \mathrm{~h}$ after transfection and the interference efficiency of siRNA1 sequence was higher than that of siRNA2 $(P<0.05) . * P<0.05, * * P<0.01$ versus the NC groups. CON, ESCC cells were cultured normally; lip2000, ESCC cells were transfected with equal amounts of Lipofectamine ${ }^{\circledR} 2000$ Reagent; NC, ESCC cell were transfected with non-targeting control siRNA as negative control .
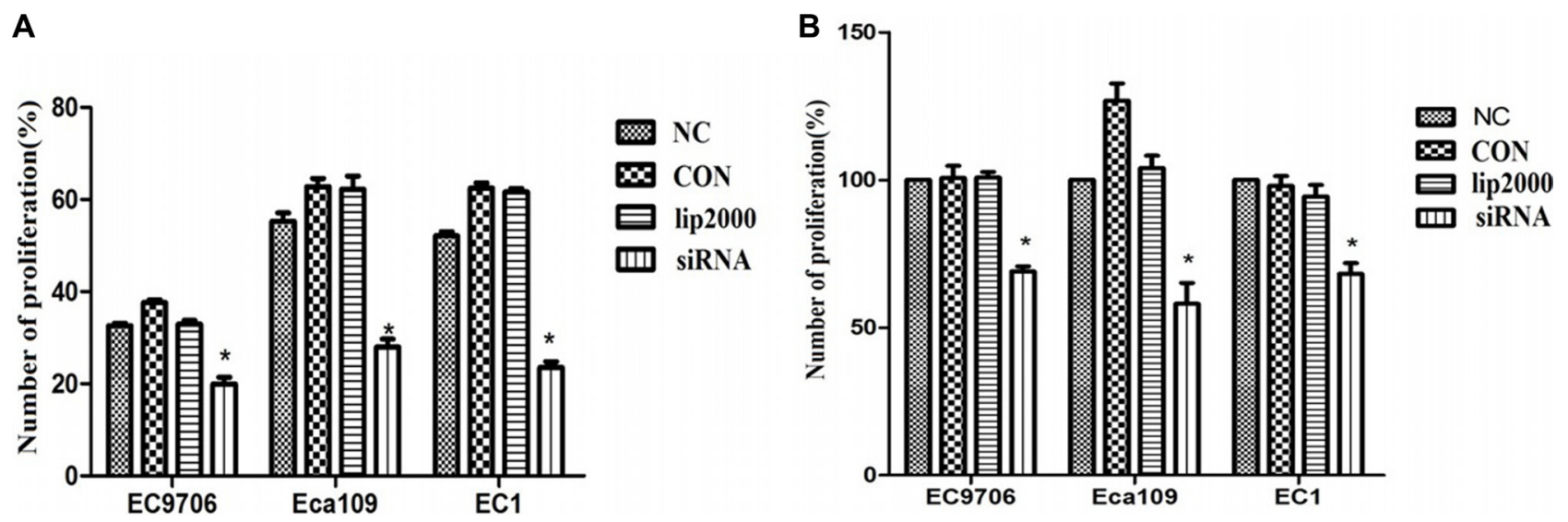

Figure 2: Proliferative rates of ESCC cells when Ets2 was knocked down. (A) the numbers of proliferative rates of siEts2EC9706, siEts2-Eca109 and siEts2-EC1 cells were $19.97 \%, 29.2 \%$ and $22.3 \%$ respectively, reducing by $40 \%, 45 \%$ and $57 \%$ compared with $\mathrm{NC}$ cells measured with EdU assay. (B) the cell survival rates of EC 9706 , Eca109 and EC1 cells were $69 \%, 58 \%$ and $68 \%$ after transfection with siEts2, reducing by $31 \%, 42 \%$ and $32 \%$ compared with NC (Figure 3B), respectively. ${ }^{*} P<0.05$. 
A NC

CON

lip2000
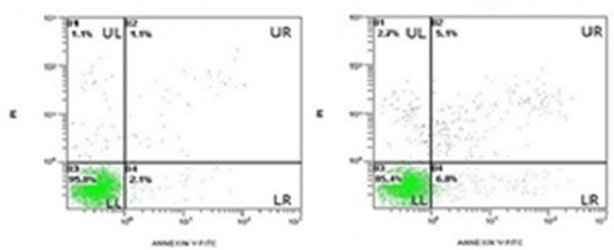

EC9706 :
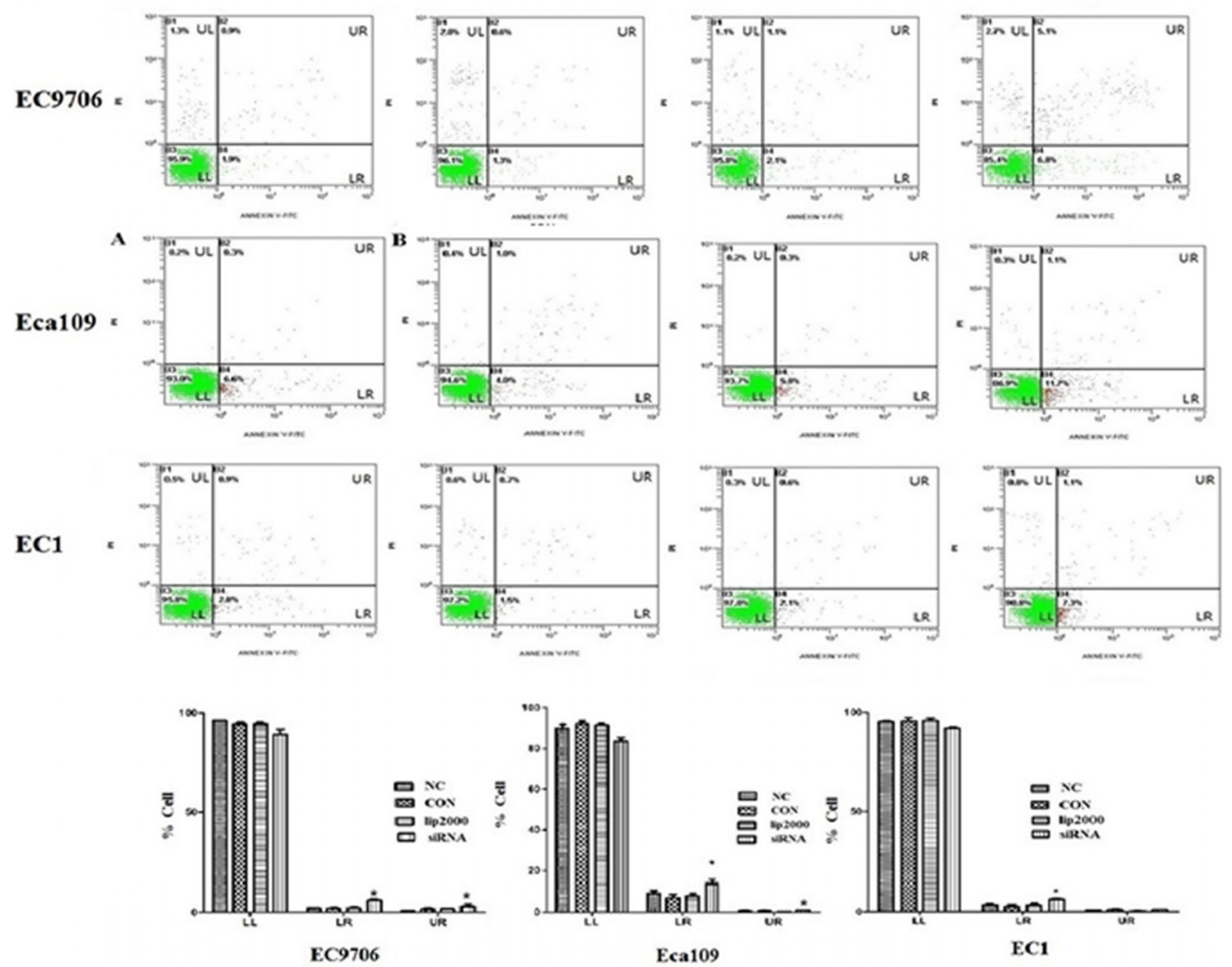

B

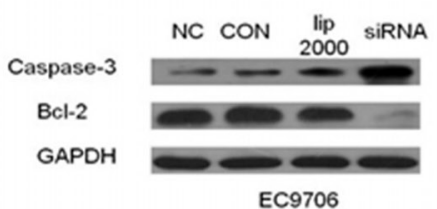

C

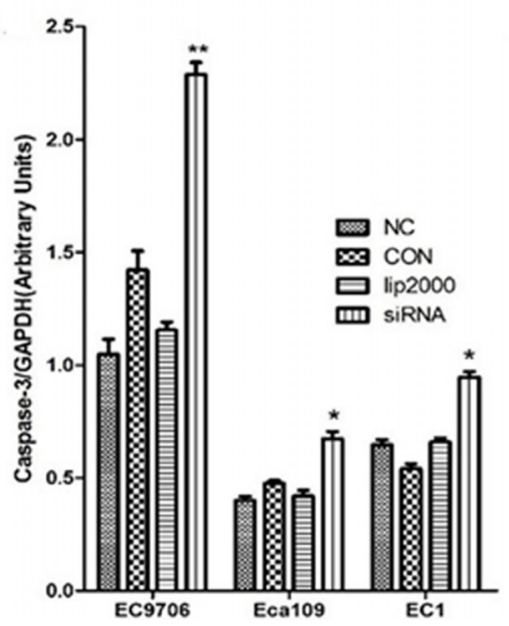

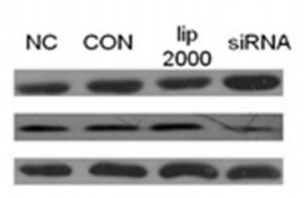

Eca109

D

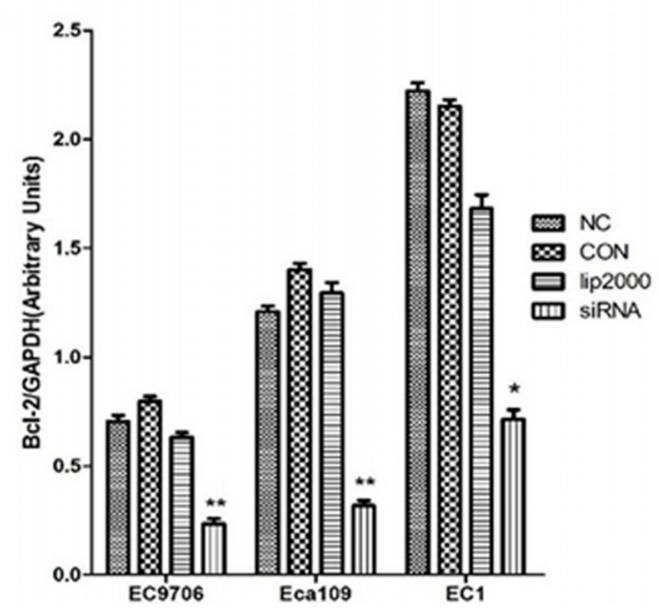

Figure 3: Ets2 knockdown induced apoptosis of ESCC cells in vitro. (A) apoptosis of ESCC cells was detected using annexin V-FITC and PI dual staining. Quadrants were designed as follows, LL: non-stained cells indicating viable cells; LR: annexin V-FITC stained cells indicating early apoptosis; UR: annexin V-FITC and PI stained cells indicating late apoptosis; and UL: PI stained cells indicating secondary necrosis. All dot plots are a representation of equal cell populations $(n=10,000)$. (B) apoptosis factor proteins caspase-3 and Bcl-2 were analyzed by Western blotting. (C) and (D) semi-quantitative analysis of caspase-3 and Bcl-2 expression. Caspase-3 was increased by $120 \%, 75 \%$ and $30 \%$ roughly in EC9706, Eca109 and EC1 cells compared with NC, while anti-apoptotic Bcl-2 protein was decreased by $70 \%, 73 \%$ and $68 \%$ in EC9706, Eca109 and EC1 cells compared with NC. $* P<0.05,{ }^{*} * P<0.01$. 


\section{Ets2 depletion induces G0/G1 period arrest in Eca109 and EC1 cells in vitro}

To explore the effect of Ets2 on cell cycle of ESCC cells, the cell cycle of ESCC cells were measured using flow cytometry after treatment with siRNA. FACScan analysis of PI-stained cells revealed that there were no significant change in cell cycle distribution of EC9706 cells compared with that in NC cells (Figure 4A, $P>0.05$ ). Nevertheless, more cells accumulated in the G0/G1 phase of the cell cycle in Eca109 and EC1 cells compared with that in NC cells (Figure 4B and 4C, $P<0.05$ ) when Ets2 was depletion, indicating Ets2 knockdown could arrest Eca109 and EC1 cells at G0/G1 phase.

\section{Ets2 depletion inhibits ESCC cells invasion}

We also investigated whether Ets2 affects the invasive ability of ESCC cells by an in vitro invasion assay. As shown in Figure 5A, knockdown of Ets2 extremely reduced the cell invasive ability. When Ets2 was interfered, the number of invaded EC9706 cells was $23.22 \pm 3.03$ compared with $58.66 \pm 5.74$ of NC cells $(P<0.05), 35.33 \pm 1.53$ compared with $54 \pm 3.61$ of NC $(P<0.05)$ of Eca109 cells and $81.33 \pm 5.85$ compared with $137 \pm 1.26$ of $\mathrm{NC}(P<0.05)$ of EC1 cells. We also examined the expression of cellular adhesion molecule, E-cadherin by western blotting in vitro and in vivo, finding that E-cadherin proteins in EC9706, Eca109 and EC1 cells rose by $54 \%, 130 \%$ and $67 \%$ respectively compared with that in NC (Figure 5B, $P<0.05$ ). Moreover, E-cadherin was also increased in Ets2-knockdown tumor tissues compared to tumor-bearing control tissues (Figure $7 \mathrm{C}$ and $7 \mathrm{D}$, $P<0.001)$. These data suggested that Ets2 silence was related to invasion inhibition of ESCC cells.

\section{Ets2 knockdown inactivates the mTOR/p70S6K signaling pathway in vitro and in vivo}

To examine the mechanistic basis of Ets2 in ESCC, we determined the effects of Ets 2 knockdown on the expression of phosphorylated mTOR (p-mTOR), p70S6K and Prdx1 of ESCC cell in vitro and cancer xenograft mouse model. Western blotting analysis showed that siRNA-mediated knockdown of Ets2 decreased the expression of p-mTOR and p-p70S6K dramatically in EC9706 Eca109 and EC1 cells and Eca109-bearing tissues as shown in Figures $6,7 \mathrm{C}$ and $7 \mathrm{D}$, indicating that Ets2 knockdown inactivated the mTOR/p70S6K signaling pathway remarkably. And Peroxiredoxin $1(\operatorname{Prdx} 1)$ which was reported associated with Ets2 was also reduced by Ets2 knockdown in ESCC cells (Figure 7C and 7D, $P<0.001$ ).

\section{DISCUSSION}

Cancer can be defined as a genetic disease, resulting as a consequence of multiple events associated with initiation, promotion and metastatic growth. Ets proteins are transcription factors that activate or repress the expression of genes, which are involved in various biological processes, including cellular proliferation, differentiation, development, transformation and apoptosis [3]. Ets2 was originally identified as a proto-oncogene, but it exhibits both tumor-promoting and -suppressive effect in different types of carcinomas. Previous studies have indicated that Ets2 factors are abnormally expressed in both tumor and stromal compartments, which frequently correlates with tumor progression. For example, Ets2 are expressed at much higher levels in neoplasias of the thyroid relative to benign and normal tissues [20]. A high Ets2 protein level in early stage of hepatocellular carcinoma has also been found [21]. Expression of Ets2 is not observed in normal colon and hyperplastic polyps, however its expression is associated with advancing tumor grade and correlates with lymph node metastasis in colon cancer [17], and Ets2 deregulated in non-small cell lung cancer was identified as one of 50 genes in mice that are involved in the progression of lung cancer from adenoma to carcinoma [22]. Previously, it was demonstrated that Ets2 expression was enhanced in ESCC tissues [18]. In the present study, we further corroborated that Ets2 was also expressed at a relatively higher level in ESCC
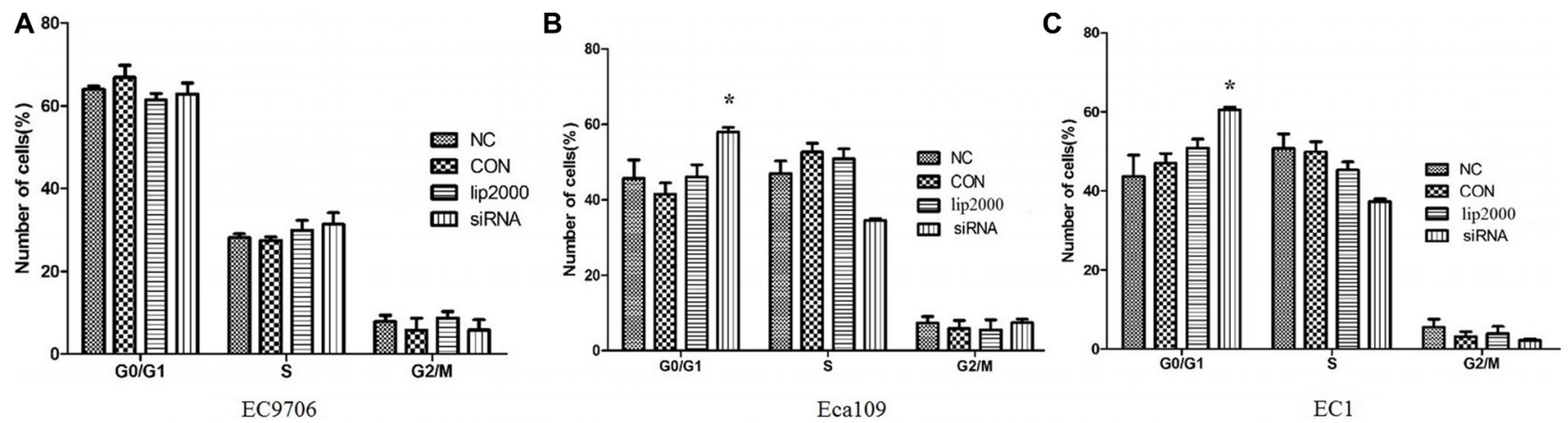

Figure 4: Effects of Ets2 knockdown on cell cycle. The number of siEts2-EC9706 cells at each cell cycle phase had no change compared with that of NC cells. The numbers of siEts2-Eca109 and siEts2-EC1 cells at G0/G1 phase were more than that of CON cells $(P<0.05) . * P<0.05$. 


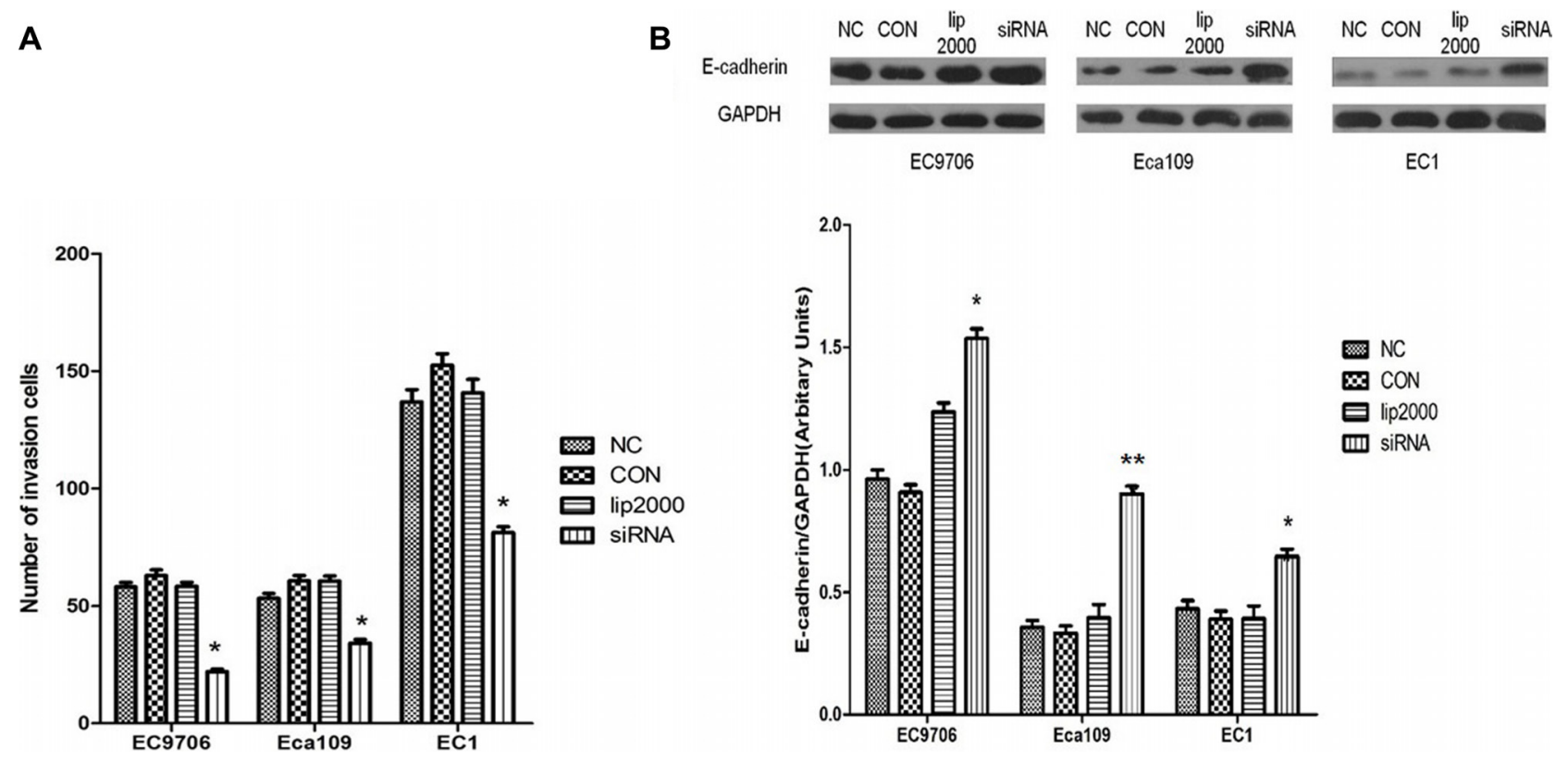

Figure 5: Inhibitory effects of Ets2 depletion on the invasive capacity of ESCC cells. (A) The results of Transwell chamber assay. The numbers of invaded siEts2-EC9706, siEts2-Eca109 and siEts2-EC1 cells were significantly reduced compared with that of invaded NC cells. (B) E-cadherin expression was detected using Western blotting and the results revealed that E-cadherin protein was significantly increased by $54 \%, 130 \%$ and $67 \%$ respectively compared with that in NC. ${ }^{*} P<0.05,{ }^{*} P<0.01$.
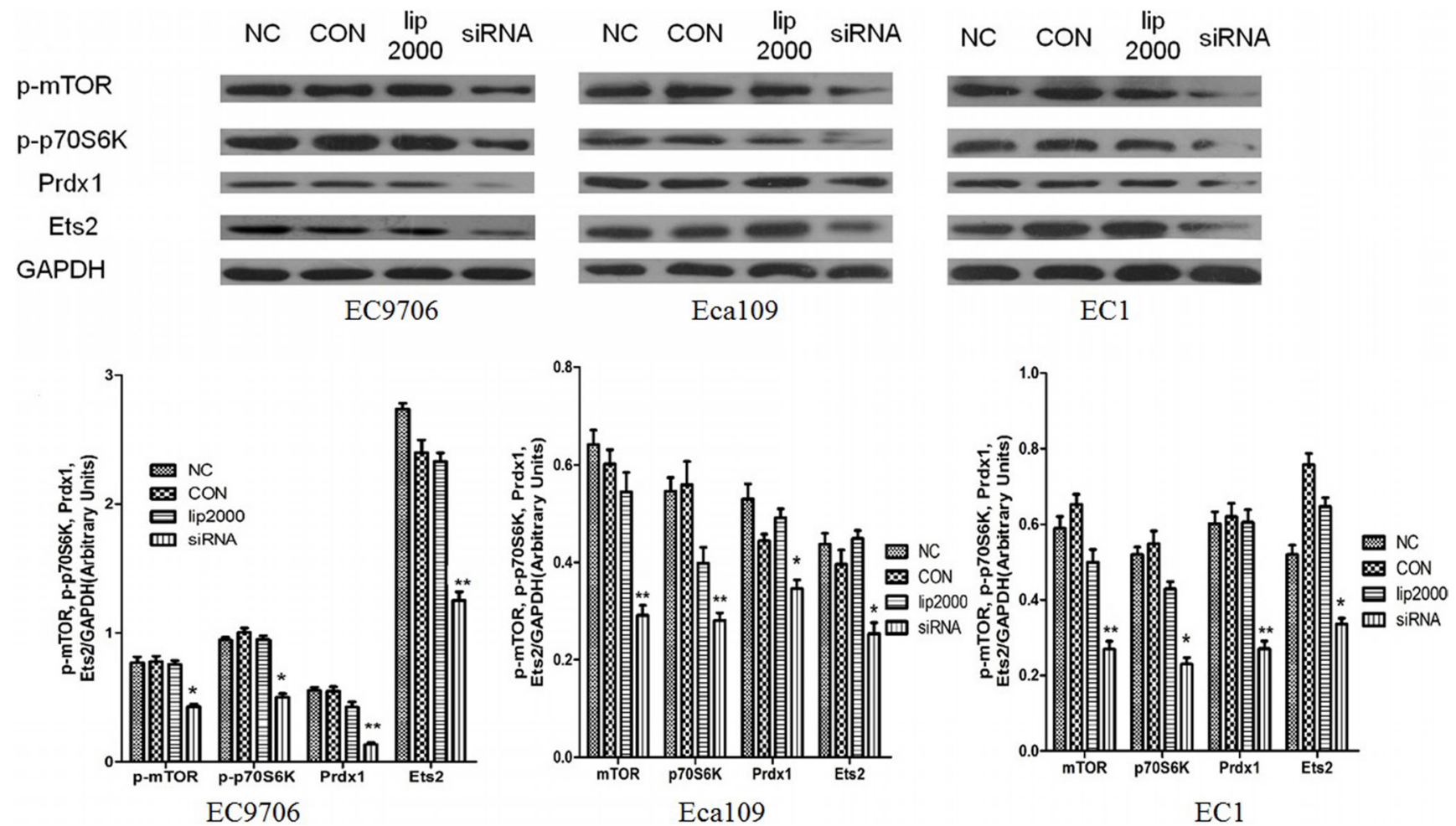

Figure 6: Effect of Ets2 knockdown on mTOR/p70S6K signaling pathway. When Ets2 was knocked down, the expression of Prdx1 protein was reduced concomitantly with the reduction of Ets2 in EC9706, Eca109 and EC1 cells $(P<0.05)$. Both p-mTOR and p-p70S6K proteins were decreased in siEts2-EC9706, siEts2-Eca109 and siEts2-EC1 cells compared with that in NC groups $(P<0.05)$. $* P<0.05, * * P<0.01$. 
cells compared to normal esophageal squamous cells. To elucidate the mechanism basis of contribution of Ets 2 to the development and progression of ESCC, we

A

B
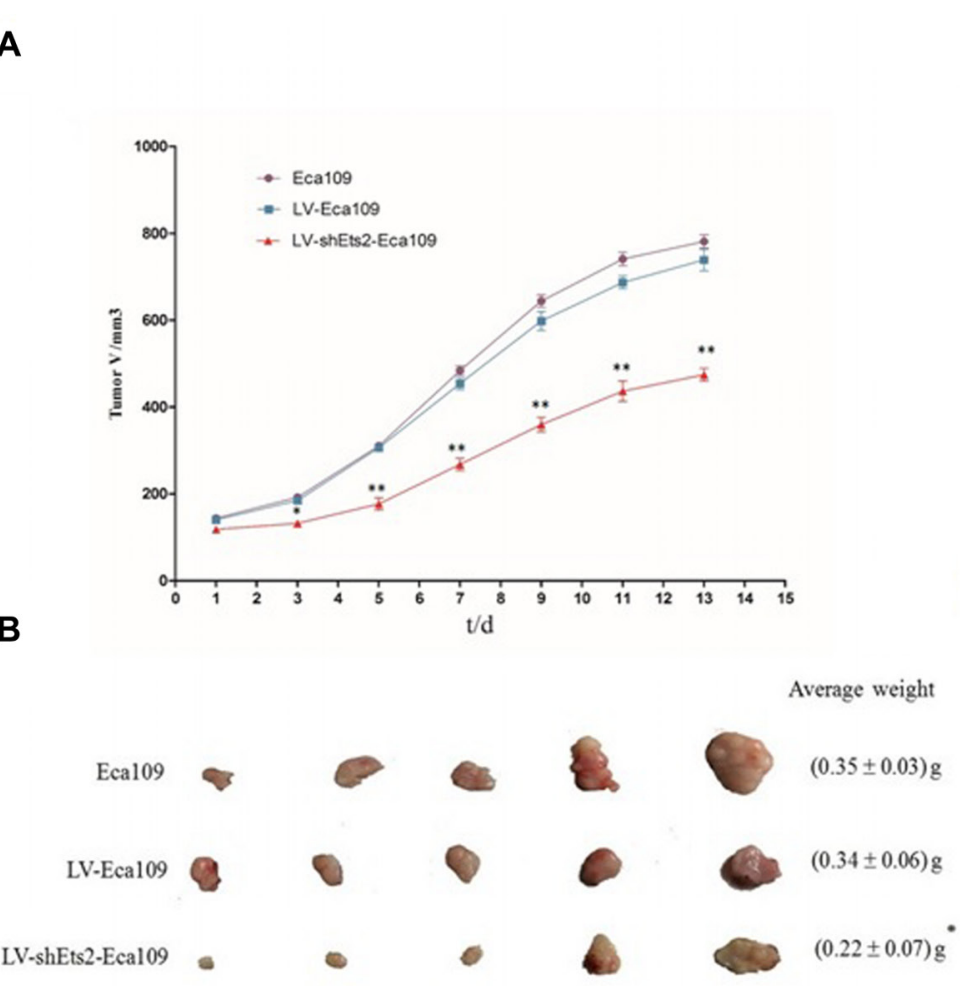

A

(n)

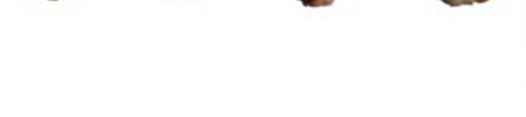

constructed an Ets2-knockdown cell model mediated by RNA interference. The most effective siRNA sequence was screened from 3 candidate sequences and Ets 2 protein c

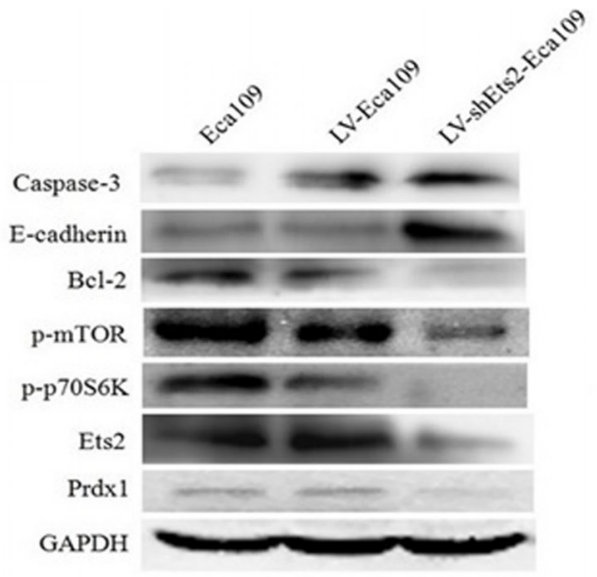

D

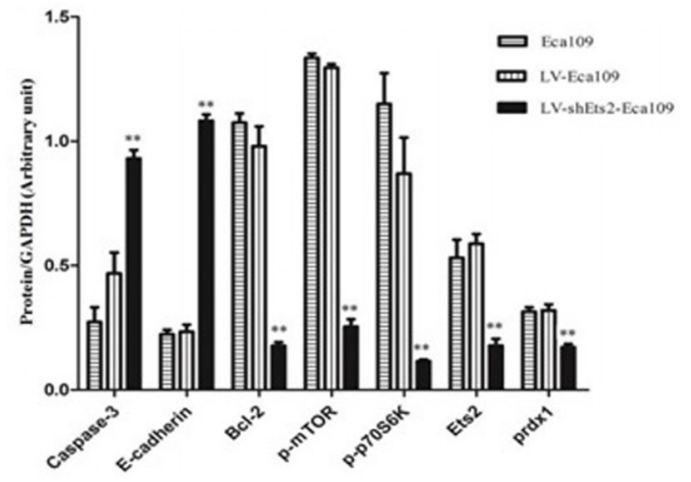

Figure 7: Effects of Ets2 silence on tumor size and protein expressions in vivo. (A) the tumor growth curve of xenograft mice. The tumor volume in LV-shEts2-Eca109 cell injected into nude mice was significantly smaller than that in LV-Eca109 and Eca109 cell-bearing mice $(P<0.001)$. (B) the tumor weight in xenograft mice. The average tumor weight in LV-shEts2-Eca109 cell-bearing mice was much lighter than that in LV-Eca109 and Eca109 cell-bearing mice $(P<0.05)$. (C) and $(\mathbf{D})$, the protein expressions were analyzed by Western blotting. Protein caspase-3 and E-cadherin were significantly enhanced in LV-shEts2-Eca109 injected mice compared with that in Eca109 and LV-Eca109 injected mice $(P<0.001)$, while the proteins of Bcl-2, p-mTOR, p-p70S6K and Prdx1 were significantly reduced as the reduction of Ets2 in xenograft mice $(P<0.001)$. $* P<0.05, * * P<0.01$.

A

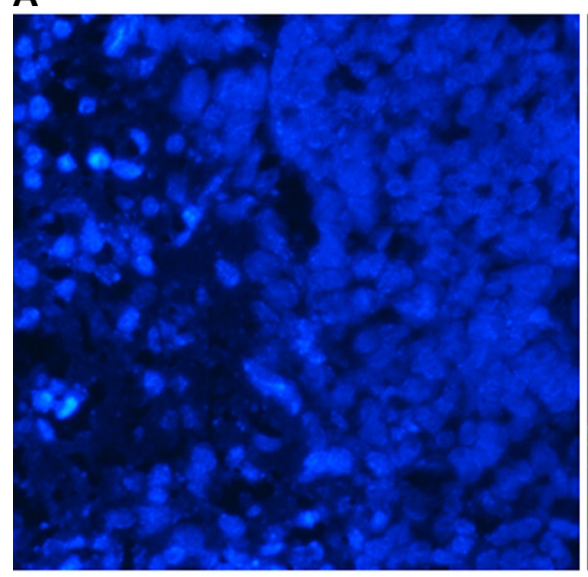

DAPI $(200 x)$
B

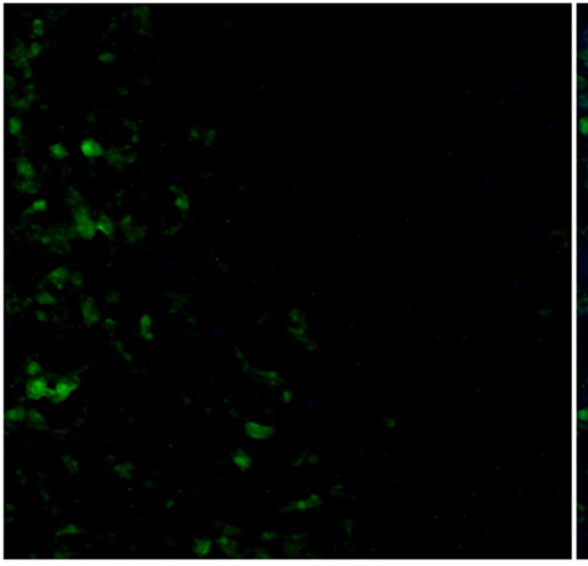

Tunel(200x)
C

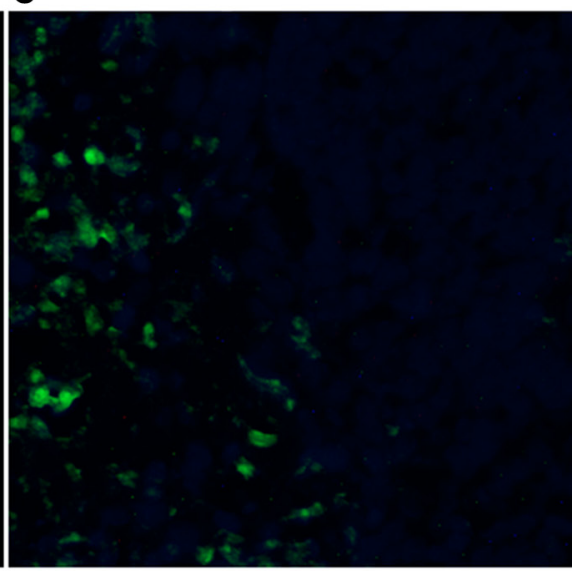

Merge(200 X )

Figure 8: Ets2 silence promotes ESCC cells apoptosis in vivo. Tunel assay demonstrated a 17.4\% apoptotic rate in Ets2 silence tissue. 
was specifically inhibited in ESCC cell lines. When Ets2 was knocked down by RNA interference, the viability and proliferation rates of ESCC cells detected by CCK8 and EdU assays were distinctly decreased in vitro. Ets2 depletion enhanced early and late stage apoptosis in EC9706 and Eca109 cells but only promoted early stage apoptosis in EC1 cells. The cell apoptosis factor caspase-3 was increased while anti-apoptotic Bcl-2 protein was decreased corresponding with the decrease in Ets2 levels. These observations suggest the presence of an intrinsic cell death pathway stimulating a wide network of signals which acts in part through the Bcl-2 family signaling cascade [23]. We also found G0/G1 period arrest in Eca109 and EC1 cells induced by Ets 2 knockdown in vitro. The number of ESCC cells that invaded Transwell chambers pre-coated with matrigel was significantly reduces and the expression of cellular adhesion molecule E-cadherin which mediates homophilic calcium dependent intracellular adhesion was enhanced after a reduction of Ets2 in vitro and in vivo, illustrating Ets2 knockdown inhibited the invasion of ESCC cells via strengthen the cellular adhesion.

Ets proteins contribute to many biological processes by control and interaction of specific target genes in various cancers. Ets2 are demonstrated to be involved in both Ras/ Raf/MAP kinase and phosphatidylinositol 3-kinase/Akt pathways in murine fibroblasts. Additionally, Ets2 has been identified as a Wnt target in colorectal cancer cells and intestinal stem cells [17]. The mammalian target of rapamycin (mTOR) is a serine/threonine protein kinase that supports cell growth, cellular metabolism, cell proliferation, cell motility, cell survival, protein synthesis, and transcription initiating angiogenesis and autophagy. Our previous study found that the mTOR/p70S6K signaling pathway was aberrantly activated in ESCC cells [24] and that the activity of $\mathrm{mTOR} / \mathrm{p} 70 \mathrm{~S} 6 \mathrm{~K}$ signaling pathway was decreased when Prdx1, a tumor-associated antigen in ESCC [25], was reduced in EC9706 cells [26]. In addition, Masaki et al. found that Ets-2 regulated the expression of Prdx1 in human prostate cancer PC3 cells [27]. Thus, we hypothesized that Ets2 could be involved in ESCC tumorigenesis through $\mathrm{mTOR} / \mathrm{p} 70 \mathrm{~S} 6 \mathrm{~K}$ signaling pathway. In our study, we found p-mTOR and p-p70S6K proteins significantly reduced and this was accompanied by the knockdown of Ets2 in vitro and in vivo, indicating that Ets2 knockdown inactivated the $\mathrm{mTOR} / \mathrm{p} 70 \mathrm{~S} 6 \mathrm{~K}$ signaling pathway.

Collectively, Ets 2 knockdown by RNA interference effectively promoted ESCC cell apoptosis, decreased ESCC cell proliferation, inhibited migration, induced G0/G1 arrest and attenuated invasion of ESCC cells in vitro and in vivo, via inactivating the $\mathrm{mTOR} / \mathrm{p} 70 \mathrm{~S} 6 \mathrm{~K}$ signaling pathway. The finding in this study could provide novel therapeutic targets and diagnostics for future ESCC therapy, as direct inhibition of Ets 2 transcription by triplex forming oligonucleotides resulted in growth inhibition and induction of apoptosis in human prostate cancer cells [28].

\section{MATERIALS AND METHODS}

\section{Cell lines and cell culture}

ESCC cell lines EC1 and Eca109 were preserved in our laboratory and EC9706 cells were provided by the State Key Laboratory of Molecular Oncology, Chinese Academy of Medical Sciences (Beijing, China). An immortalized normal esophageal epithelial cell line Het-1A was a gift of Prof. Zhenyu Ji (Henan Academy of Medical and Pharmaceutical Sciences, China). STR analysis was performed to ensure the cells are tumor cells (Supplementary material). Cells were cultured in RPMI 1640 medium (HyClone Laboratories, USA) supplemented with $10 \%$ fetal bovine serum (FBS) (TBD Science, China), at $37^{\circ} \mathrm{C}$ in the presence of $5 \% \mathrm{CO} 2$.

\section{Ets2 knockdown by RNA interference}

Three candidate siRNA targeting sequences against Ets2 (siEts2) were synthesized by Shanghai GenePharma Co., Ltd (Shanghai, China) after our design. The silencing effect of the siRNA was then evaluated by Western blotting. For assays in vivo, the most effective target sequence was ligated into lentiviral pFH-L plasmid (Shanghai Genechem), packaged in HEK-293T cells and then lentivirus particles of Ets2 (LV-shEts2) were collected with lentivirus particles of scramble shRNA as a negative control (LV). Then, lentivirus particles of siEts2 and scramble shRNA were added to the culture medium to infect Eca109 cells (marked as LV-shEts2-Eca109 and LVEca109). After lentivirus infection, the cells were washed with PBS and collected to inject into athymic nude mice.

\section{Western blotting analysis}

The total proteins of ESCC cells and tissues were extracted with Total Protein Extraction (Sangon Biotech, China). The protein extracts were separated with SDSPAGE after their concentrations were determined using Bradford protein assay kit (Sangon Biotech, China) and then transferred to polyvinylidine difluoride microporous (PVDF) membranes (Millipore, USA). The blotted membranes were treated with $5 \%(\mathrm{w} / \mathrm{v})$ skimmed milk in TBST buffer $(10 \mathrm{mmol} / \mathrm{L}$ Tris, $150 \mathrm{mmol} / \mathrm{L} \mathrm{NaCl}$, and $0.2 \%$ Tween 20), and incubated for $1 \mathrm{~h}$ at room temperature with primary antibody anti Ets2 (Santa Cruz Biotechnology, USA), p-p70S6K (Cell Signaling Technology, USA), Peroxiredoxin 1 (Cell Signaling Technology, USA) and caspase-3 (Abcam, USA), E-cadherin (Abcam, USA) and GAPDH (Cell Signaling Technology, USA). They were then washed and incubated for $1 \mathrm{~h}$ with a horseradish peroxidase-linked anti-rabbit secondary antibody (Sangon Biotech, China). Finally, the bands of specific proteins on the membranes were examined by Enhanced Chemiluminescence Kit (Beijing ComWin Biotech Co.,Ltd, 
China) and densitometric analysis was performed with Image J software (NIH).

\section{Cell proliferation assay in vitro}

Cell proliferation was measured by the 5-ethynyl-2'deoxyuridine (EdU) assay according to the manufacturer's instructions. Briefly, $5 \times 10^{5}$ cells/well were seeded in a 96-well flat-bottomed plate, grown at $37^{\circ} \mathrm{C}$ for $24 \mathrm{~h}$, and then exposed to $50 \mu \mathrm{M}$ of EdU for additional $2 \mathrm{~h}$ at $37^{\circ} \mathrm{C}$. Cells were fixed with $4 \%$ formaldehyde for $15 \mathrm{~min}$ and permeabilized with $0.5 \%$ Triton X-100 for 20 min. After being rinsed three times with PBS, the cells were treated with ApolloR reaction cocktail followed by DNA staining with Hoechst 33342 for 30 min for visualizing under fluorescent microscope (Nikon, Japan).

\section{Cell apoptosis assay in vitro and in vivo}

Apoptotic cells were determined with an Annexin V-FITC cell apoptosis kit (BD Biosciences, USA) according to the manufacturer's protocol. Briefly, the transfected ESCC cells were washed with PBS and subsequently incubated for $15 \mathrm{~min}$ at room temperature in the dark in $100 \mu \mathrm{l} 1 \times$ binding buffer containing $5 \mu \mathrm{l}$ Annexin V-FITC and $5 \mu \mathrm{l}$ propidiumiodide (PI). Apoptosis was determined by fluorescence microscope (Zeiss Axio Observer A) and FACScan laser flow cytometer (FACSCalibur, Becton Dickinson, USA).

We used the In situ cell death detection kit according to the manufacturer's instructions for the TUNEL assay. Briefly, $5 \mu \mathrm{m}$-paraffin sections were made from the center of the paraffin-embedded tumor tissues, and the sections were deparaffinized, washed with xylene, ethanol and PBS. Each section was incubated with $20 \mu \mathrm{g} / \mathrm{mL}$ proteinase $\mathrm{K}$ solution at $37^{\circ} \mathrm{C}$ for $30 \mathrm{~min}$ after permeation in $1 \%$ triton $\mathrm{X}-100$ and blocking in $3 \% \mathrm{H}_{2} \mathrm{O}_{2}$. TUNEL staining was performed using the Dead End fluorometric TUNEL system (KeyGEN BioTECH, China) according to the manufacturer's protocol.

\section{Transwell chamber assay for invasion in vitro}

Transwell chambers pre-coated with matrigel (Corning-Costar, USA) were used to detect invasion capacity of siRNA-transfected EC9706, Eca109 and EC1 cells. Firstly, serum-free RPMI 1640 medium was planted into the upper of a 24-well Transwell plate with polycarbonate membrane filters containing $8-\mu \mathrm{m}$ pores and then Transwell plate was incubated for $1.5 \mathrm{~h}$ in the cell incubator hydrating the extracellular matrix (ECM) membrane. After transfection for $48 \mathrm{~h}$, the $300 \mu \mathrm{l}$ cell suspension with $2 \times 10^{5}$ cells $/ \mathrm{ml}$ were planted into the upper compartment and the lower compartment of the Transwell plate contained $500 \mu \mathrm{l}$ of RPMI1640 medium with $5 \%$ FBS. After an 8 h-incubation at $37^{\circ} \mathrm{C}$, noninvading cells were gently removed using a cotton-tipped swab. The cells were then fixed with methanol for $10 \mathrm{~min}$ followed by $0.2 \%$ crystal violet staining. The number of invaded cells in 10 distinct randomly selected fields was counted under light microscope.

\section{In vitro cell cycle distribution analysis}

EC9706, Eca109, and EC1 cells transfected with siRNA and control cells were respectively harvested, fixed in $70 \%$ ice-cold ethanol overnight at $4{ }^{\circ} \mathrm{C}$, and then stained with PI. Cell cycle analysis was carried out by flow cytometry (BD, USA).Establishment of Eca109 Cell Xenograft Mouse model In Vivo

A total of 15 pathogen-free male BALB/C athymic nude mice were purchased from VITAL RIVEA Laboratory Animal Co. Limited (Beijing, China). The 6-week old mice were divided into 3 groups after acclimatization for 7 days, each group was injected with $2 \times 10^{6}$ Eca109, LV-shEts2-Eca109 and LV-Eca109 cells, respectively. After 7 days, the tumor length (long axis) and tumor width (short axis) of each tumor-bearing mouse were measured every other day for 2 weeks, and tumor volumes were calculated as $1 / 2 \times$ length $\times$ width $^{2}$ $\left(\mathrm{mm}^{3}\right)$ according to previous method [19]. Mice were then sacrificed under deep anesthesia and the final volumes, as well as the weights of the tumors were measured. The tumor inhibition rate was calculated as (tumor weight in control mice-tumor weight in treated mice)/tumor weight in control mice $\times 100$. Eventually, the tumor tissues were kept in liquid nitrogen for further investigations.

\section{Statistical analysis}

All experiments were carried out in triplicate, and the experimental results were analyzed using a SPSS software package (version 17.0). All numerical data were expressed as means \pm standard deviation (SD). ANOVA was used for multiple comparisons with a value of $P<0.05$ considered to be statistically significant. All $P$ values were generated from two-sided tests for statistical significance.

\section{ACKNOWLEDGMENTS AND FUNDING}

This work was supported by Innovative Research Team (in Science and Technology) of the University in Henan Province [15IRTSTHN022], Plan for Scientific Innovation Talent of Henan Province [154200510008] and Key Research Project of Higher Education of Henan Province [15A310028 and 15A180022], The International Collaboration Research Program for Talents of Henan Province [2016GH15].

\section{CONFLICTS OF INTEREST}

These authors declare that there is no conflicts of interests concerning this paper. 


\section{REFERENCES}

1. Hou G, Zhang Q, Wang L, Liu M, Wang J, Xue L. mTOR inhibitor rapamycin alone or combined with cisplatin inhibits growth of esophageal squamous cell carcinoma in nude mice. Cancer Lett. 2010; 290 :248-54. doi: 10.1016/j. canlet.2009.09.015.

2. Kim T, Grobmyer SR, Smith R, Ben-David K, Ang D, Vogel SB, Hochwald SN. Esophageal cancer-the five year survivors. J Surg Oncol. 2011; 103 :179-83. doi: 10.1002/ jso. 21784 .

3. Seth A, Watson DK. ETS transcription factors and their emerging roles in human cancer. Eur J Cancer. 2005; 41:2462-78.

4. Sharrocks AD. The ETS-domain transcription factor family. Nat Rev Mol Cell Biol. 2001; 2:827-37.

5. Kasten M, Giordano A. Cdk10, a Cdc2-related kinase, associates with the Ets 2 transcription factor and modulates its transactivation activity. Oncogene. 2001; 20:1832-38.

6. Sevilla L, Aperlo C, Dulic V, Chambard JC, Boutonnet C, Pasquier O, Pognonec P, Boulukos KE. The Ets2 transcription factor inhibits apoptosis induced by colonystimulating factor 1 deprivation of macrophages through a Bcl-xL-dependent mechanism. Mol Cell Biol. 1999; 19:2624-34.

7. de Nigris F, Mega T, Berger N, Barone MV, Santoro M, Viglietto G, Verde P, Fusco A. Induction of ETS-1 and ETS-2 transcription factors is required for thyroid cell transformation. Cancer Res. 2001; 61:2267-75.

8. Sementchenko VI, Schweinfest CW, Papas TS, Watson DK. ETS2 function is required to maintain the transformed state of human prostate cancer cells. Oncogene. 1998; 17:2883-88.

9. Santoro A, Maggio A, Carbone P, Mirto S, Caronia F, Acuto S. Amplification of ETS2 oncogene in acute nonlymphoblastic leukaemia with $\mathrm{t}(6 ; 21 ; 18)$. Cancer Genet Cytogenet. 1992; 58: 71-75.

10. Buggy Y, Maguire TM, McDermott E, Hill AD, O’Higgins N, Duffy MJ. Ets2 transcription factor in normal and neoplastic human breast tissue. Eur J Cancer. 2006; 42:485-91.

11. Simpson S, Woodworth CD, DiPaolo JA. Altered expression of Erg and Ets-2 transcription factors is associated with genetic changes at 21q22.2-22.3 in immortal and cervical carcinoma cell lines. Oncogene. 1997; 14:2149-57.

12. Liu AY, Corey E, Vessella RL, Lange PH, True LD, Huang GM, Nelson PS, Hood L. Identification of differentially expressed prostate genes: increased expression of transcription factor ETS-2 in prostate cancer. Prostate. 1997; 30:145-53.

13. De la Houssaye G, Vieira V, Masson C, Beermann F, Dufier JL, Menasche M, Abitbol. M ETS-1 and ETS-2 are upregulated in a transgenic mouse model of pigmented ocular neoplasm. Molecular Vision. 2008; 14:1912-28.

14. Kabbout M, Garcia MM, Fujimoto J, Liu DD, Woods D, Chow CW, Mendoza G, Momin AA, James BP, Solis L,
Behrens C, Lee JJ,Wistuba II, et al. ETS2 mediated tumor suppressive function and MET oncogene inhibition in human non-small cell lung cancer. Clin Cancer Res 2013; 19 :3383-95. doi: 10.1158/1078-0432.CCR-13-0341.

15. Wasylyk B, Hagman J, Gutierrez-Hartmann A. Ets transcription factors: nuclear effectors of the Ras-MAPkinase signaling pathway. Trends Biochem Sci. 1998; 23:213-16.

16. Smith JL, Schaffner AE, Hofmeister JK, Hartman M, Wei G, Forsthoefel D, Hume DA, Ostrowski MC. Ets-2 is a target for an akt (Protein kinase B)/jun N-terminal kinase signaling pathway in macrophages of motheaten-viable mutant mice. Mol Cell Biol. 2000; 20:8026-34.

17. orge Múnera, Cecena G, Jedlicka P, Wankell M, Oshima RG. Ets2 regulates colonic stem cells and sensitivity to tumorigenesis. Stem Cells. 2011; 29 :430-39. doi: 10.1002/ stem.599.

18. Li X, Lu JY, Zhao LQ, Wang XQ, Liu GL, Liu Z, Zhou CN, Wu M, Liu ZH. Overexpression of ETS2 in human esophageal squamous cell carcinoma. World J Gastroenterol. 2003; 9:205-08.

19. Park JC, Lee YJ, Choi HY, Shin YK, Kim JD, Ku SK. In Vivo and In Vitro antitumor effects of platycodin d, a saponin purified from platycodi radix on the H520 lung cancer cell. Evid Based Complement Alternat Med. 2014; 13. doi: 10.1155/2014/478653.

20. Findlay VJ1, LaRue AC, Turner DP, Watson PM, Watson DK. Understanding the role of ETS-mediated gene regulation in complex biological processes. Adv Cancer Res. 2013; 119:1-61. doi: 10.1016/B978-0-12-4071902.00001-0.

21. Ito Y, Miyoshi E, Takeda T, Nagano H, Sakon M, Noda K, Tsujimoto M, Monden M, Matsuura N. Linkage of elevated ets-2 expression to hepatocarcinogenesis. Anticancer Res. 2002; 22:2385-89.

22. Bonner AE, Lemon WJ, Devereux TR, Lubet RA, You M. Molecular profiling of mouse lung tumours: association with tumour progression, lung development, and human lung adenocarcinomas. Oncogene. 2004; 23: 1166-76.

23. Haupt S, Berger M, Goldberg Z, Haupt Y. Apoptosis-the p53 network. J Cell Sci. 2003; 116: 4077-85.

24. Hou G, Xue L, Lu Z, Fan T, Tian F, Xue Y. An activated $\mathrm{mTOR} / \mathrm{p} 70 \mathrm{~S} 6 \mathrm{~K}$ signaling pathway in esophageal squamous cell carcinoma cell lines and inhibition of the pathway by rapamycin and siRNA against mTOR. Cancer Lett. 2007; 253:236-48.

25. Ren P, Ye H, Dai L, Liu M, Liu X, Chai Y, Shao Q, Li Y, Lei N, Peng B, Yao W, Zhang J. Peroxiredoxin 1 is a tumor-associated antigen in esophageal squamous cell carcinoma. Oncol Rep. 2013; 30 :2297-303. doi: 10.3892/ or.2013.2714.

26. Gong F, Hou G, Liu H, Zhang M. Peroxiredoxin 1 promotes tumorigenesis through regulating the activity of mTOR/ p70S6K pathway in esophageal squamous cell carcinoma. Med Oncol. 2015; 32 :455. doi: 10.1007/s12032-014-0455-0. 
27. Shiota M, Izumi H, Miyamoto N, Onitsuka T, Kashiwagi E, Kidani A, Hirano G, Takahashi M, Ono M, Kuwano M, Naito S, Sasaguri Y,Kohno K.Ets regulates peroxiredoxin1 and 5 expressions through their interaction with the highmobility group protein B1. Cancer Sci. 2008; 99 :1950-59. doi: 10.1111/j.1349-7006.2008.00912.x.
28. Carbone GM, Napoli S, Valentini A, Cavalli F, Watson DK, Catapano CV. Triplex DNAmediated downregulation of Ets2 expression results in growth inhibition and apoptosis in human prostate cancer cells. Nucleic Acids Res 2004; 32: 4358-67. 